\title{
Super-high Speed, Accuracy, and Modularized Residue Number System based on Redundant Binary Representation
}

\author{
Tsugio Nakamura* ${ }^{*}$ Member \\ Kazuhiro Abe ${ }^{* *}$ Non-member \\ Narito Fuyutsume ${ }^{* * *}$ Member \\ Hiroshi Kasahara** ${ }^{* * *}$ Member \\ Teruo Tanaka*** Member
}

\begin{abstract}
The multiplier and divider used for specific hardware of public key cryptosystem arithmetic are constructed from many adders and subtractors to improve the accuracy of the key. However, with the increase of accuracy, the propagation delay problem becomes unavoidable. Although some paper have proposed that a divider using redundant binary representation is effective to cope with this problem, no considerations were given to the problems of rounding error and accuracy of the remainder. This paper proposes a method, based on inherent bit sliced architecture, that can cope with these problems and that is expandable to any level of accuracy. It is expected to make it applicable to hardware for public key cryptosystem that can be flexible in coping with the expansion of the key string and the variable length key.
\end{abstract}

Keywords: Modularizing, Residue Number System, Redundant Binary Representation, Public Key Cryptosystem, Divider.

\section{Introduction}

High-speed and high-precision operation mechanism is required of arithmetic units such as addition, subtraction, multiplication, division, etc. in real-time systems performing signal processing operation for speech, image as well as for many generic industrial technologies. The implementation of this mechanism shows that sometime the hardware can cope with the operations better than the software.

In the case of adder and subtractor as fundamental digital arithmetical operation, a propagation delay increases in proportion to the bit length of the operation because a carry and borrow propagate with the increase in the precision. A carry look ahead (CLA) is also a high-speed operation, but is unsuitable for high precision operations and precision expandability by bit slice implementation, furthermore, a CLA cannot be expected a remarkable speed-up because of the propagation delay between the chips, likely to be cascaded. A solution to this propagation problem can be expected to use redundant binary representation with constant

\footnotetext{
* Department of Information network, Kokusai Junior College Egota 4-15-1, Nakano, Tokyo, 165-0022

** Fujitsu LTD.,

Tokyo

*** The School of Information Environment, Tokyo Denki University

Muzai Gakuendai 2-1200, Inzai-shi, Chiba, 270-1382

**** The Department of Electrical Engineering, Tokyo Denki University

Nishiki-chou 2-2, Kanda, Chiyoda, Tokyo 101-8457
}

delay, regardless of the bit length of the operation, as proposed by Avizeinis ${ }^{(1)}$.

The redundant binary representation, another highspeed operation, is a type of Singed Digit (SD) representation and brings about an increase in the number of the terminals and in the size of the circuit because one digit must be represented by at least two bits (and it is this fact that limited its implementation in the integrated circuit technology of the previous generation). Recent years have seen significant advances in this field with a marked tendency toward a shift from system board implemented on a printed circuit board to System on Chip (SOC) that can be implemented on a single chip ${ }^{(2)}$. Based on this fact, we can expect the implementation of digital system with very high levels of functions and SOC of more than 10 mega-gate. Lately, attention has been also given to arithmetic units that use high-speed redundant binary representation technology, even though it brings about an increase in the scale of the circuit ${ }^{(3)(4)}$. However, with a conventional divider using redundant binary representation a rounding error is often introduced into the quotient without taking the remainder into consideration. In addition, a conventional divider needs to normalize a partial remainder at each step, and it cannot perform divisions beyond its operational accuracy even by one bit. A precision expandability beyond this level of accuracy has not been developed until now.

In this paper we propose a new divider architecture as a solution to the problems posed by a conventional divider. For the implementation of this new divider it is necessary, in order to decide the sign of the partial 
remainder, to check the bits within the MSB to LSB interval. To this end, a sign decision circuit is added to every parallel subtractor. This will result in the divider performing an operation with an n-bit unsigned binary which eliminate the need for normaliziation at every step. This type of division does not require to perform any specific data-conversions and, in addition, it can produce an accurate remainder with no rounding error. The new divider architecture is similar to previously studied architectures ${ }^{(9)(16)}$, and it allows full scalability, a bit slice implementation and modularity. Now, however, with the use of redundant binary representation we can address problems of modularity and extension of the integration scale. Consequently, it can be applied easily to RSA public key cryptosystem processing the residue arithmetic with a variable-length key to strengthen the security. Until now, various residue arithmetic mechanisms have been developed for RSA public key cryptosystem ${ }^{(5)-(7)}$ without, however, proposing considerations for the modularizing the arithmetic units. Moreover, to the best of our knowledge, no one else has proposed an architecture that can be applied to arithmetic unit with numbers of arbitrary bit length simply by cascading identical units.

With regard to integrated circuits such as SOC, it is difficult to design all circuit blocks as single-supplier cores (macro cells), therefore the chip integration occurs by using cores supplied by several manufacturers. In SOCs, reusable cores are so required and, for example, bit slice implementation of arithmetic units can be effectively achieved. Until recently it was impossible to increase the division accuracy by even one bit and divisions operations could cope with the arithmetic only by adding modules by bit slice implementation. By using the method we propose, division can be performed efficiently with cascaded modules suitable for operational precision and using clock cycles by bit slice implementation. In addition, we expect this method to lead to a simplification of design and testing, to reduce cost, to simplify the performing of arithmetic functions with a variable-length precision and to improve the reliability by small scale implementation.

This paper is not the proposal of the new theory on Residue Number System(RNS), but the proposal on the realization of high-speed RNS with a modularizing scheme that is expandable to any level of accuracy by cascading a number of the same modules.

\section{Redundant Binary Representation and Addition/Subtraction Method}

The reminder of this section will describe the redundant binary representation used with a divider and its computation rules.

Generally, a n-digit binary integer is represented as:

$$
\left(a_{n-1} a_{n-2} \cdots a_{0}\right)_{2} \cdots \cdots \cdots \cdots \cdots \cdots \cdots \cdots
$$

This is represented in decimal numbers as:

$$
\left(\sum_{i=0}^{n-1} a_{i} 2^{i}\right)_{10}
$$

In normal binary numbers, the coefficient $a_{i}$ of the $i$-th digit has two values $\{0,1\}$ but in a redundant binary representation, which is a kind of SD representation with the base- 2 number system, the $a_{i}$ has three values $\{\overline{1}, 0,1\}$, where $\overline{1}$ represents -1 . It is necessary for each digit to represent the coefficients with at least two bits ${ }^{(4)(8)}$. In this paper, for each digit the value $\overline{1}$ consists of two bits $\{1,0\}$, the value 0 consists of two bits $\{0,0\}$ and the value 1 consists of two bits $\{0,1\}$, respectively. A redundant binary adder consists of cascaded full adders suited to the required bit length as in the case of normal binary adder.

A redundant binary addition operation is done as per the following two steps:

(1) Determination of the intermediate sum and carry at the $i$-th digit and $(i-1)$ th digit.

(2) Addition of the intermediate sum to the carry.

In redundant binary adder with three value $\{\overline{1}, 0,1\}$ the addition is done at constant time because a carry propagation to the $(i+1)$ th digit is inhibited at the $i$-th digit based on its computation rules. Table 1 shows the computation rule in redundant binary addition without carry propagation. The intermediate sum $\left(s_{i}\right)$ and carry $\left(c_{i}\right)$ are determined under the augend $\left(x_{i}\right)$, addend $\left(y_{i}\right)$ and $x_{i-1} ; y_{i-1}$ of the $(i-1)$ th digit.

A redundant binary subtractor, where the reversal of the sign is obtained by reversing each digit, operates similarly to a redundant binary adder. The subtraction result of $A-B$ is obtained by the addition of $A$ to $\bar{B}$ which is $B$ with each digit reversed. Therefore, simi-

Table 1. Computation rule in redundant binary

\begin{tabular}{|c|c|c|c|c|}
\hline \multirow{3}{*}{$\begin{array}{c}\text { Augend } \\
x_{i} \\
\end{array}$} & \multirow{3}{*}{$\begin{array}{c}\text { Addend } \\
y_{i}\end{array}$} & \multirow{3}{*}{$\begin{array}{l}\text { one lower digit } \\
\quad x_{i-1}, y_{i-1}\end{array}$} & \multicolumn{2}{|c|}{ intermediate } \\
\hline & & & carry & sum \\
\hline & & & $c_{i}$ & $s_{i}$ \\
\hline 1 & 1 & $*$ & 1 & 0 \\
\hline 0 & 1 & either is negative & 0 & 1 \\
\hline 1 & 0 & neither is negative & 1 & $\overline{1}$ \\
\hline 0 & 0 & \multirow{3}{*}{$\log _{1}$} & \multirow{3}{*}{0} & \multirow{3}{*}{0} \\
\hline 1 & $\overline{1}$ & & & \\
\hline$\overline{1}$ & 1 & & & \\
\hline$\overline{1}$ & 0 & either is negative & $\overline{1}$ & 1 \\
\hline 0 & $\overline{1}$ & neither is negative & 0 & $\overline{1}$ \\
\hline$\overline{1}$ & $\overline{1}$ & $*$ & $\overline{1}$ & 0 \\
\hline
\end{tabular}
addition without carry propagation.

Table 2. Computation rule in redundant binary

\begin{tabular}{|c|c|c|c|c|}
\hline minuend & subtra- & one lower & inter & mediate \\
\hline$x_{i}$ & $\begin{array}{r}\text { hend } \\
y_{i} \\
\end{array}$ & $\begin{array}{l}\text { digit } \\
x_{i-1}, y_{i-1} \\
\end{array}$ & $\begin{array}{c}\text { borrow } \\
b_{i} \\
\end{array}$ & $\begin{array}{c}\text { difference } \\
d_{i} \\
\end{array}$ \\
\hline 1 & $\overline{1}$ & $*$ & 1 & 0 \\
\hline 0 & $\overline{1}$ & $\begin{array}{l}x_{i-1}=1 \text { or } \\
y_{i-1}=\overline{1}\end{array}$ & 1 & $\overline{1}$ \\
\hline 1 & 0 & otherwise & 0 & 1 \\
\hline 0 & 0 & \multirow[b]{2}{*}{ * } & \multirow[b]{2}{*}{0} & \multirow[b]{2}{*}{0} \\
\hline $\begin{array}{l}1 \\
\overline{1}\end{array}$ & $\begin{array}{l}1 \\
\overline{1}\end{array}$ & & & \\
\hline$\overline{1}$ & 0 & $\begin{array}{l}x_{i-1}=1 \text { or } \\
y_{i-1}=\overline{1}\end{array}$ & 0 & $\overline{1}$ \\
\hline 0 & 1 & otherwise & $\overline{1}$ & 1 \\
\hline$\overline{1}$ & 1 & $*$ & $\overline{1}$ & 0 \\
\hline
\end{tabular}
subtraction without carry propagation. 
larly to addition, a redundant binary subtraction will always reduce the carry propagation to one digit ${ }^{(8)}$. Table 2 shows the computation rule in redundant binary subtraction without carry propagation.

\section{New Proposed Divider}

A divider consists of several subtractors. In this section we propose a new approach to make up a parallel subtractor using sign decision circuits.

3.1 Conventional Division Algorithm As a division algorithm using SD representation, the SRT (Sweeney, Robertson and Tocher) algorithm (a type of nonrestoring division) is well known. With this algorithm the divisor and partial remainder are needed to normalize and adjust the digit position at each step and the algorithm is generally used only for bits of fixed length.

To offset such limitations, a subtraction-shift method with restoring division without normalizing is adopted for the divider we are proposing. Using our method, modularization or bit slice implementation can be easily realized and we can obtain an accurate quotient and a remainder. However, because the bit length of the dividend is longer than that of the divisor, subtractions carried out by cascading $\mathrm{n}$-digit assigned divisors cannot be easily realized due to the difficulty of cascading divisor, subtractors and latch circuits. The n-digit assigned divisor module was made possible by changing the algorithm as follows. A n-digit assigned divisor and the subtractor are fixed in each module and the cascaded dividends, shifted by one-bit, perform the subtraction. We call this method a modified subtraction-shift method. We have developed some modular divider using the modified subtraction-shift method with normal binary numbers ${ }^{(9)(10)}$ and the divider is implemented on a special purpose RSA public key cryptosystem LSI ${ }^{(11)}$.

A process of $n$-digit division based on the modified subtraction-shift method is shown in Fig.1, and an example of division is shown in Fig.2, where $R$ is a partial remainder and it becomes the reminder in the last step of the process. $R$ ' is a temporary partial remainder and its value will determine if it will become a new reminder or not. In Fig. 1 divisible? denotes the process of deciding whether or not $R$ ' is divisible in the middle of the operation. If $R$ ' is divisible, the operation ends there because there is no longer need for the operation. The symbol \& denotes the combination of the $x_{i-1}$ bit with the binary value of $R$ after a one-bit left shift. As shown in the example in Fig.2, if we have a dividend $X=1101$, a divisor $Y=0101$ will give, after four subtractions and three shift operations a quotient $Q=0010$ and a remainder $R=0011$.

3.2 Parallel Subtractor Structure The ndigit parallel divider is made of $\mathrm{n} n$-digit subtractor arrays shifted individually by one digit, while a $n$-digit sequential type divider is composed of a n-digit parallel subtractor operating $\mathrm{n}$ times, in repetition. In this section we examine the $\mathrm{n}$-digit parallel subtractor that makes up a divider.

The process of deciding $R^{\prime} \geq 0$ in Fig. 1 is different

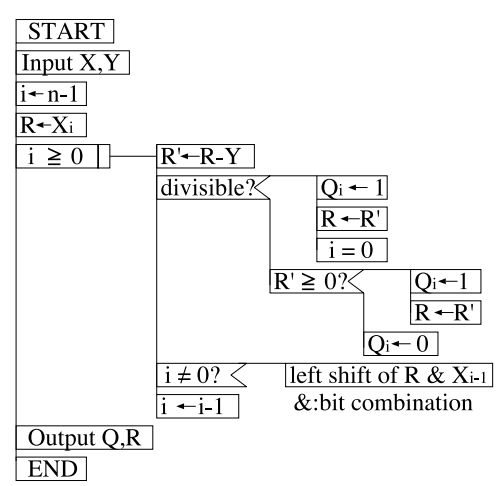

Fig. 1. A process of n-digit division.

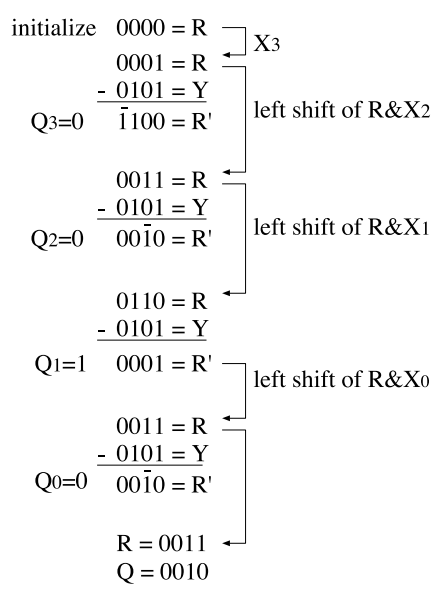

Fig. 2. An example of division.

from that of normal binary numbers. In the case of normal binary numbers the sign of the subtraction result is determined only by the highest borrow. This is because, if the subtraction result is positive, the highest borrow of the subtractor is surely " 0 ", and if the result is negative, then it is surely " 1 ". However, in case of redundant binary representation, the borrow propagates, at the most, by only one digit, and the borrow is not always determined by the most significant digit, even if it is negative. Consequently, the sign of the subtraction result must be determined by the result itself at each step and the operation for the sign determination should progress from the most significant digit to the least significant digit but only until the first digit carrying a sign. The sign decision circuit for the subtraction result is therefore implemented in each subtractor. We will then obtain that each dividend, divisor and quotient - except the remainder - are represented by unsigned binary number and it is not necessary to normalize them during the operation. This constitutes the sign decision circuit structure we are proposing ${ }^{(12)(13)}$.

The structure of parallel subtractor that have implemented the sign decision circuit for the subtraction result is shown in Fig.3, and a subtractor cell structure is shown in Fig.4, where the input signals of one lower digit(explained in section 2) are omitted and will not be further considered. In this method the result $(d)$, obtained by subtracting the divisor $(y)$ from the partial remainder $(x)$, is inputted to the sign decision circuit. If 


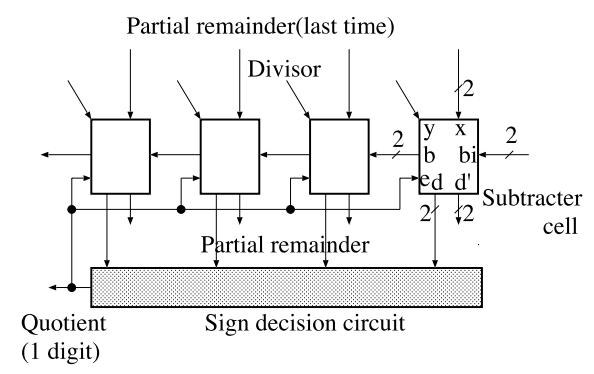

Fig. 3. Parallel subtractor structure.

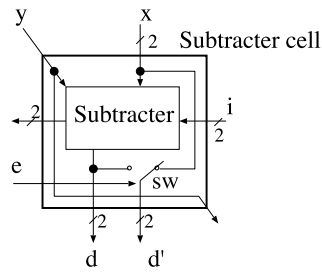

Fig. 4. Subtractor cell structure.

the subtraction result is positive, the output of the circuit and $Q_{i}$ are both equal to "1", and the temporary partial reminder becomes a true one. In that case the switch $(S W)$ will also change from being connected to the cell input to its output $\left(d^{\prime} \leftarrow d\right)$. In case of negative result, the output $\left(Q_{i}\right)$ of the sign decision circuit is " 0 " and the temporary partial remainder is invalid. The switch $(S W)$ in this case will be required to form a connection $\left(d^{\prime} \leftarrow x\right)$ with the cell input, but only in case its position had been otherwise.

The division algorithm with normal binary numbers (see section 3.1) is applied to the divider by adding the sign decision circuit with the parallel subtractor. The subtraction result is decided between a positive and a negative value by the sign decision circuit. The quotient of the result is outputted as a normal binary number because the circuit outputs a result either as " 0 " or " 1 " and the remainder is outputted as redundant binary representation.

3.3 Sign Decision Circuit Structure The function of the sign decision circuit is to discriminate the sign of the subtraction result with redundant binary representation, that is to discriminate " $R^{\prime} \geq 0$ " as shown in Fig1. The subtraction redundant binary result is examined from the most significant digit, and the sign is assigned when the first non-zero digit is encountered. If the value is " 1 ", the sign is positive, and if " $\overline{1}$ " is negative, and if all digits are " 0 ", then the result is zero.

The structure of the proposed divider is as follows. The subtraction result with redundant binary representation is separated into a positive bit string $\mathrm{A}$ and a negative bit string $\mathrm{B}$ and both of them are compared by a magnitude comparator. The bit string $\mathrm{A}$ is composed by the lower bits of all digits which form the redundant binary representation of the subtraction result, and the bit string $B$ is made of the upper bits of the same digits. When the A and B strings are compared, if $\mathrm{A}$ is greater than $\mathrm{B}$, the subtraction result is positive, otherwise it is negative or, alternatively, nil.

Although it would have been possible to use a tra-

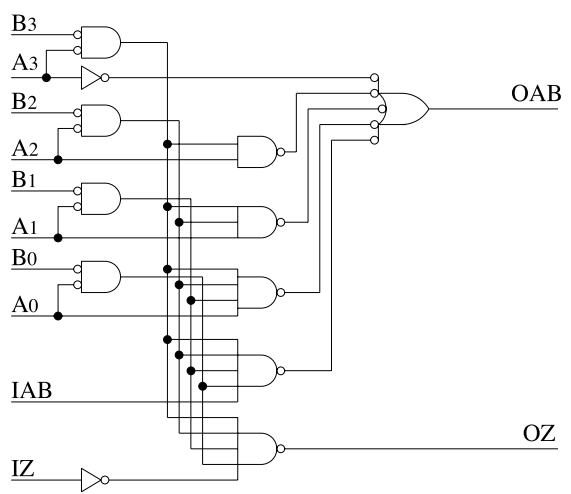

Fig. 5. Sign decision circuit structure.

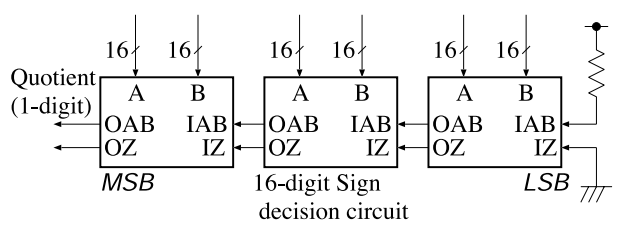

Fig. 6. Cascade connection of sign decision circuit.

ditional magnitude comparator in the sign decision circuit, we propose some improvements that will reduce the gate count and operating time of a standard comparator. A magnitude comparator(for example the 4-bit magnitude comparator 74LS85) usually has three outputs $A>B ; A=B$ and $A<B$. The proposed sign decision circuit has two outputs, $A=B$ (based on whether the inputs were divisible or not) and $A<B$ (used to determine the sign of the partial remainder). The output $A>B$ is not necessary. An additional advantage is found in the fact that the value " 1 " cannot be simultaneously in both $A_{i}$ and $B_{i}$ strings and so the circuit can be simplified by substituting a NOR gate for an Exclusive OR gate which detects $A_{i}=B_{i}$. The simplified 4-digit sign decision circuit is shown in Fig.5. Here we have that: if it is $A \geq B$, it is $O A B=1$; and if it is $A<B$, it is $O A B=0$; and if it is $A=B$, it is $O Z=0$, which denotes the result of divisible operation. $I A B$ and $O A B$ of the sign decision circuits are cascaded to perform expanded division with parallel subtractor and, similarly, $I Z$ and $O Z$ are cascaded. An example of three cascaded 16-digit sign decision circuits is shown in Fig.6.

\subsection{Use Of The Parallel Subtractor In A} Divider Circuit

3.4.1 Use With A Parallel Divider The ndigit parallel divider consists of $n$ n-digit subtractor arrays shifted progressively of one digit, as the structure shown in Fig.7. After subtracting a divisor from a partial remainder in a subtractor array, new partial remainders are inputted to the next array, as selected by the subtraction results. The division result is obtained from the $\mathrm{n}$-digit subtractions executed $\mathrm{n}$ times ${ }^{(10)}$. In this structure, $\mathrm{n}$ n-digit sign decision circuits with n n-digit subtractor arrays are considered necessary.

In case of a m-times cascaded replication of the n-digit divider (allowing an operational precision of the parallel divider of $[m \times n])$, one-digit quotient is obtained by 


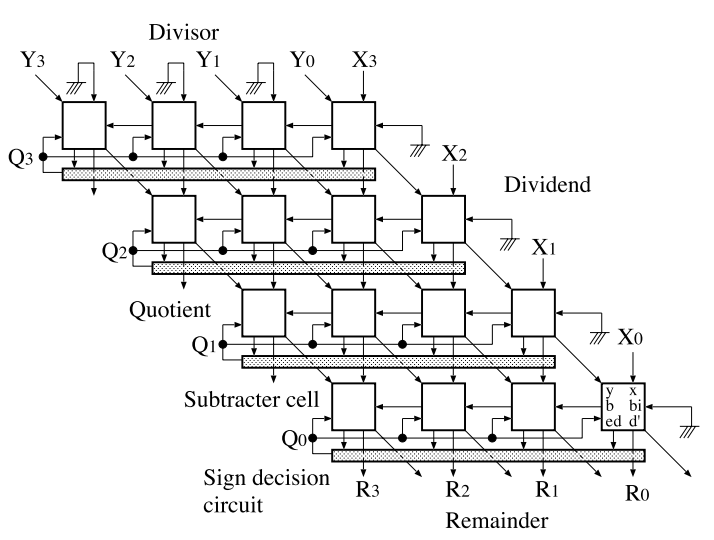

Fig. 7. 4-bit parallel divider structure.

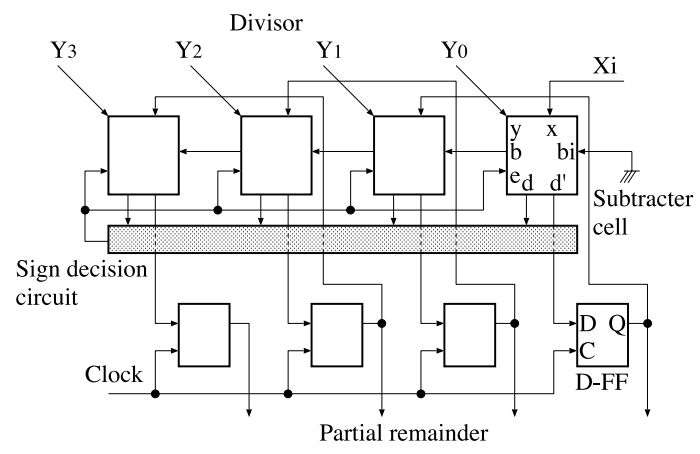

Fig. 8. 4-bit sequential divider structure.

a m-replication of the subtractor. To obtain a m-digit quotient, it is necessary to replicate $\mathrm{m}$-times the process required for a one-digit quotient. To increase the accuracy of the divider we can simply arrange many similar modules in a two-dimensional array, although the array will require $m^{2}$ modules.

3.4.2 Use with a Sequential Type Divider The mechanism of a sequential type divider is such that the value of one-digit quotient $Q_{i}$ is obtained, at every clock cycle, from the upper digit. The structure is shown in Fig.8. A parallel divider is made of subtractor arrays shifted progressively one digit, as mentioned above, but with a sequential type divider that require only one subtractor array the bit shift operation is synchronized by clock pulse and therefore only one sign decision circuit is needed per module. The number of the subtractor arrays can be reduced with the sequential type divider by re-using the same subtractor array under a synchronized clock pulse. In order to obtain one-digit quotient in case of $\mathrm{m}$ times expansions (as many as the operational precision of the sequential type divider), m subtractor arrays are necessary and therefore $\mathrm{m}$ divider modules are cascaded to perform a division expanded $m$ times, as many times as operational precision. From the viewpoint of operation speed, the parallel divider is much faster than the sequential type although the number of the modules increases with the increase in the precision. Taking expandability into consideration, the sequential type is more suitable to the task. So we have proposed a quasiparallel multiplier/divider structure with normal binary numbers which features the high speed processing of the
Table 3. Gate counts of the divider.

\begin{tabular}{c|r}
\hline circuit block & gate count \\
\hline redundant binary subtractor & 416 \\
\hline sign decision circuit & 162 \\
\hline multiplexer & 128 \\
\hline Flip-Flop for shift and latch & 224 \\
\hline $\begin{array}{c}\text { circuit for converting the division } \\
\text { result to normal binary numbers }\end{array}$ & 144 \\
\hline
\end{tabular}

parallel type structure and the expandability of the sequential type ${ }^{(14)-(16)}$. This method is easily applicable to the quasi-parallel divider too.

\section{Performance Evaluation}

In this section, we discuss the performance of the proposed divider, of the carry propagation type divider, of the CLA type divider and of a conventional divider with redundant binary representation. We will assume that the operational precision is expanded by $\mathrm{m}$ cascaded modules, and that the operational precision in one sequential type module is n-digit. The sequential carry propagation type divider that we previously developed is used as reference because a divider architecture with a feasible distributed processing has not, so far, ever been realized. A conventional divider with redundant binary representation cannot be modular, consequently, a case of integration on one chip will be presented here. The gate count and delay time for the basic gate NAND, NOR and NOT are 1 gate, $1 \Delta$ respectively ${ }^{(17)}$.

4.1 Gate Count The gate counts calculated for one 16-digit module in the proposed divider are given in Table 3. The equation for calculating the gate count of $\mathrm{m}$ modules with $\mathrm{n}$-digit is given in (3). The subtractors in this divider acts to subtract normal binary numbers from redundant binary numbers and the result is obtained in redundant binary numbers because the dividend (partial remainder) is in redundant binary numbers and the divisor is in normal binary numbers. The gate count of subtractor, multiplexer and the circuit for converting the division result to normal binary numbers are proportional to their digit length. However, although the gate count of the sign decision circuit is not proportional to its digit length, for ease of explanation, it is assumed that the gate count is proportional to it and therefore the gate count per one digit is one-sixteenth of the scale of a 16-digit sign decision circuit.

$$
\begin{aligned}
\text { gatecounts }= & (416+162 \\
& +128+224+144 / 16 \times \mathrm{mn} \\
= & 67.13 \mathrm{mn} \ldots \ldots \ldots \ldots \ldots \ldots \ldots)
\end{aligned}
$$

The result of a similar calculation for the gate count of carry propagation type divider is $20 \mathrm{mn}+2 \mathrm{~m}$, that of CLA type divider is $78.06 \mathrm{mn}+2 \mathrm{~m}$ and that of a conventional redundant binary number type divider is $69 \mathrm{mn}+13$ giving consideration to the need for some adders and subtractors. On the assumption that these four type of dividers are implemented in a super-high accuracy operation system (i.e. a public key cryptosystem), the comparison of the gate counts for 128-digit $(\mathrm{m}=8)$ and for 1,024-digit $(\mathrm{m}=64)$ modules ex- 
Table 4. comparison of gate count.

\begin{tabular}{c|c|c}
\hline & \multicolumn{1}{|c|}{128 -digit } & \multicolumn{1}{c}{1,024 -digit } \\
\hline proposed method & $8,592(1.00)$ & $68,741(1.00)$ \\
\hline carry propagation type & $2,576(0.30)$ & $20,608(0.30)$ \\
\hline CLA type & $10,008(1.16)$ & $80,064(1.16)$ \\
\hline $\begin{array}{c}\text { conventional } \\
\text { redundant binary }\end{array}$ & $8,848(1.03)$ & $70,669(1.03)$ \\
\hline
\end{tabular}

Table 5. Propagation delay time of the divider.

\begin{tabular}{c|r}
\hline circuit block & delay time $[\Delta]$ \\
\hline redundant binary subtractor & 5 \\
\hline sign decision circuit & $2 \mathrm{~m}+1$ \\
\hline multiplexer & 3 \\
\hline $\begin{array}{c}\text { circuit for converting the division } \\
\text { result to normal binary numbers }\end{array}$ & $3 \mathrm{mn}$ \\
\hline
\end{tabular}

panded by only cascaded dividers whose operational precision is 16-digit per module is shown in Table 4 . The values shown between parenthesis in the Table 4 denote the target rates for the proposed method.

4.2 Propagation Delay Time The propagation delay time for each circuit block in the proposed divider is shown in Table 5 . We assume that each time margin to trigger latch and shift circuits is $10 \%$ of the maximum delay which occurs in the sign decision circuit. The subtractor, the sign decision circuit and the multiplexer are used mn times for each dividion operation, so the maximum propagation delay time is given by (4) below. Depending on which digit from the most significant digits the sign decision of the subtraction result is based upon, the delay time will vary. Though highly improbable, the maximum delay time is calculated on the assumption that the sign is decided in the least significant digit, every time, for all steps of the subtraction.

$$
\begin{aligned}
\delta= & (5+2 \mathrm{~m}+1+3 \\
& +0.1 \times 2 \mathrm{~m} \times 2) \mathrm{mn}+3 \mathrm{mn} \\
= & (2.4 \mathrm{~m}+12) \mathrm{mn}[\Delta] \cdots \cdots
\end{aligned}
$$

Equations for other types of dividers are obtained in a similar fashion. The propagation delay time of the subtractor in the carry propagation type divider is proportional to the operational digit length, but the delay time is given as $(3.6 \mathrm{mn}+4.8 \mathrm{~m}+3) \mathrm{mn}[\Delta]$ because the circuits for the sign decision and for converting the division result to normal binary numbers are not necessary in the carry propagation type. In the CLA type divider the propagation delay time is given as $(4.8 \mathrm{~m}+3) \mathrm{mn}[\Delta]$. Finally, in the conventional redundant binary numbers type divider, the delay time is obtained as $28 \mathrm{mn}[\Delta]$ in consideration of the constant delay time and regardless of the operational digit length of the sign decision circuit. This, however, takes in no account the delay time for normalizing.

On the assumption that these four type of dividers are implemented on one module, the comparison of the propagation delay time for 128 -digit $(\mathrm{m}=8)$ and 1,024 $\operatorname{digit}(\mathrm{m}=64)$, expanded only by cascaded dividers whose operational precision is 16-digit per one module, is shown in Table 6 . The values in parenthesis in the table
Table 6. Comparison of propagation delay time.

\begin{tabular}{c|r|r}
\hline & \multicolumn{1}{|c|}{128 -digit } & \multicolumn{1}{c}{1,024 -digit } \\
\hline proposed method & $3,994(1.00)$ & $169,574(1.00)$ \\
\hline carry propatation type & $64,281(16.1)$ & $4,092,518(24.1)$ \\
\hline CLA type & $5,299(1.33)$ & $317,645(1.87)$ \\
\hline $\begin{array}{c}\text { conventional } \\
\text { redundant binary }\end{array}$ & $3,584(0.90)$ & $28,672(0.17)$ \\
\hline
\end{tabular}

\begin{tabular}{|c|c|c|}
\hline $\mathrm{I} / \mathrm{O}$ & signal name & number of signals \\
\hline I & dividend & $\mathrm{n}$ \\
\hline I & divisor & $\mathrm{n}$ \\
\hline I & borrow & 2 \\
\hline I & one lower digit & $\overline{2}$ \\
\hline I & multiplexer control & 1 \\
\hline $\mathrm{I}$ & $\begin{array}{l}\text { quotient(dividend latch } \\
\text { \& sift circuits) }\end{array}$ & 1 \\
\hline I & $\begin{array}{l}\text { quotiont(subtractor \& } \\
\text { sift circuits) }\end{array}$ & 1 \\
\hline I & $\begin{array}{l}\text { most significant digit of } \\
\text { subtractor \& shift circuits }\end{array}$ & 1 \\
\hline I & $\begin{array}{l}\text { cascade for the sign } \\
\text { decision circuits }\end{array}$ & 2 \\
\hline \multirow[t]{2}{*}{ I } & $\begin{array}{l}\text { contraol for clock } \\
\qquad \& \text { others }\end{array}$ & 5 \\
\hline & total & $2 n+15$ \\
\hline $\mathrm{O}$ & $\begin{array}{l}\text { quotient \& devidend } \\
\text { shift circuit }\end{array}$ & $\overline{n+1}$ \\
\hline $\mathrm{O}$ & remainder & $2 \mathrm{n}$ \\
\hline $\mathrm{O}$ & borrow & 2 \\
\hline \multirow[t]{2}{*}{$\mathrm{O}$} & $\begin{array}{l}\text { cascade for the sign } \\
\text { decision circuits }\end{array}$ & 2 \\
\hline & total & $3 n+5$ \\
\hline & total of I/O signals & $5 n+20$ \\
\hline
\end{tabular}

Table 7. Number of I/O signals of sequential divider.

denote the proposed method target rates.

4.3 Number of Signals Usually a bus structure is introduced for input-output data, however, for the case of architecture whose I/O signals are separate (i.e. when it is not a bus structure), each of their relational expressions, except for the power supply terminal, is shown in Table 7. Similarly, the total number of $\mathrm{I} / \mathrm{O}$ signals in the divider with normal binary numbers is given as $4 n+13$. I/O signals usually should be multiplexed actually because a bus structure is introduced for I/O data: therefore each total of the signals decreases for that.

\section{Comparison}

The comparison of the proposed type of divider with the normal binary type, the CLA type and the conventional type is presented in this section.

First, it should be noted that the divider of the proposed method provides a performance 16 times faster than the subtraction-shift type which performs a normal binary integer division. But this type of the proposed divider is slower than a conventional divider without bit sliced architecture with redundant binary representation under the influence of the sign decision circuit. That shows a tendency to increase the propagation delay in the longer digit length. Although in the conventional type it is necessary to normalize the divisor and the remainder at every step, the delay is not included in Table 
6. Consequently, in order to normalize every step, the narrowing of the different delay time between proposed method type and conventional redundant binary numbers type is considered here. The maximum delay time for the proposed divider was calculated on the assumption that the sign of the subtraction result was decided by the least significant digit at every step, that is highly improbable. On the basis of a technical report in which the sign is decided, in most cases, by the upper digit ${ }^{(3)}$, the calculated operation time can be expected to have a much faster operation.

Second, the proposed method type is better than CLA type on the circuit scale and propagation delay time. That is so when considering that the sign decision circuits of the proposed divider are composed of simpler circuits than that in the CLA type.

Thirdly, the asynchronous divider in reference ${ }^{(3)}$, though it is impossible to decide whether in operation it would be divisible, it easily suits the proposed method and is applicable to all of numeric value.

As a last instance, the conventional divider with redundant binary representation is characterized by the divisor and partial remainder need for normalizing and it does not have a bit sliced architecture because it performs only fixed-length arithmetic; on the other hand, with proposed method a rounding error is not introduced into the quotient and is applicable to normal carry propagation type easily and is possible to realize the bit slice implementation easily too. An efficient division is performed with cascaded modules suitable for operational precision and with clock cycles by bit slice implementation. In addition, it is expected that the design and test will be simplified and of low cost, that the performing of the arithmetic with a variable-length precision be easy and an improvement of the reliability by small scale implementation.

The divider architecture proposed here in consideration of the bit slice implementation provides more hardware flexibility and expandability and therefore the proposed method is considered an architecture suitable for SOC because of their tendency toward larger scale integration.

\section{Conclusions}

In this paper we have proposed that the method of divider architecture with redundant binary representation is easily applicable to subtraction-shift method and that it can achieve an accurate quotient and a remainder applicable to super-high accuracy exponentiation for public key cryptosystem that needs accurate remainders. In performance evaluation, the proposed divider should be compared to others to assess, for any bit length, the scale of the circuit and the operation speed. However, such comparisons cannot be easily carried out because architerctures similar to the one proposed could not be identified. In this paper we have therefore considered relevant a comparison between four methods. Although all the methods discussed show outstanding characteristics, it cannot be easily concluded, on the basis of circuit scale and propagation delay time, which one of the methods assessed is the best one because each method has unique characteristics of utilization. Comparisons made with three other methods are summarized here:

(1) the conventional redundant binary representation type has high speed performances and is characterized by an operational digit length that does not influences operation time;

(2) the carry propagation type allows the smallest circuit scale realization;

(3) the CLA type shows high speed characteristic by processing the carry propagation circuits in parallel.

The method we propose can be expected to provide a performance 16 times faster than the subtraction-shift type with normal binary numbers without bit sliced architecture. Our method is also better than the CLA type from the circuit scale and propagation delay time point of view; on the other hand, the conventional redundant binary representation type performs fixed-length arithmetic at highest speed, although it can not be used beyond its operational precision. Also, though the proposed type is not faster than the conventional type, it can easily expand its operational precision to any precision level using the proposed architecture because of its original bit sliced architecture.

Finally, the realization of the bit spliced divider, which is the relevant characteristic of this method, is expected to reduce costs and to make it applicable to hardware for public key cryptosystem that can be flexible in coping with the expansion of the key string and the variable length key. Furthermore, this architecture is suitable for SOC for which the re-use of the core is required.

(Manuscript received April 16, 2004, revised Jan. 27, 2005)

\section{References}

(1) A. Avizeinis: "Signed Digit Number Representations for Fast Parallel Arithmetic", IRE Trans. Electronic Comput., Vol.KC-10, No.9, pp.389-400, Sept. (1961)

(2) H.M. Birnbaum: "How VSIA Answers the SOC Dilemma", IEEE Computer, pp.42-50, Jun (1999)

(3) E. Nakano, M. Imai, H. Nakamura, and T. Nanya: "Fast Asynchronous Nonrestoring Division with Signed Digit Representation", Technical Report of IEICE of Japan, ICD99-9, CPSY99-9, FTS99-9, pp.21-28, April (1999)

(4) N. Takagi, H. Yasuura, and S. Yajima: "A VLSI-Oriented High-Speed Divider Using Redundant Binary Representation", Trans. IEICE of Japan, Vol.J67-D, No.4, pp.450-457 (1984-4)

(5) S. Ishii, K. Ohyama, and K. Tanaka: "2040-bit High-Speed Modulus Processor for Public Key Encryption", Trans. IEICE of Japan, Vol.J82-D-I, No.4, pp.571-580 (1999-4)

(6) IBM Japan: "1024-bit RSA Encryption Accelerator", NIKKEI BYTE, pp.154-155 (1999-2)

(7) SafeNet, Inc: "Embedded IPTM-21 Exponentiation Accelerator", www.safenet-inc.com, May (2002)

(8) M. Saito, M. Hinosugi, Y. Tsunekawa, and M. Miura: "Structure Method of High-Speed Redundant Binary AdderSubtractor Representing Each Digit By Hybrid 2 Bits/3 Bits", Technical Report of IEICE of Japan, CAS99-41, VLD99-41, DSP99-57, pp.83-90 (1999-6)

(9) T. Nakamura and H. Kasahara: "A Proposal of Easily Cascadable Divider for any Word Length", Trans. IEE of Japan, Vol.111-C, No.3, pp.123-128 (1991-3) 
(10) T. Nakamura and H. Kasahara: "An Architecture for Parallel Divider of any Word Length numbers", Trans. IEICE of Japan, Vol.J75-C-II, No.5, pp.185-192 (1992-5)

(11) T. Nakamura, H. Ohishi, and H. Kasahara: "A Bit Sliced System on RSA Public Key Cryptosystem Implementation", Trans. IEE of Japan, Vol.118-C, No.7/8, pp.1073-1081 (1998-7/8)

(12) K. Abe, H. Kasahara, and T. Nakamura: "A Divider With no rounding errer for Numbers of Arbitrary Word Length Using Redundant Binary Representation", Joint Elect. Conv. Kansai Chap., G13-8, Nov (1999)

(13) K. Abe, H. Kasahara, and T. Nakamura: "Chip Sliced Divider Using Redundant Binary Representation", Technical Report of IEICE of Japan, CPM99-123, ICD99-219, pp.23-29, Dec (1999)

(14) T. Nakamura and H. Kasahara: "A Proposal of Quasi-Parallel Divider for Numbers of Arbitrary Word Length and its Application for VSI", Technical Report of IEICE of Japan, CAS9940, VLD99-40, DSP99-56, pp.75-82, Jun (1999)

(15) T. Nakamura, T. Sato, N. Suzukawa, T. Saitou, K. Abe, and H. Kasahara: "A Proposal of Quasi-Parallel Multiplier/Divider for Numbers of Arbitrary Word Length and its Application for RSA Public Key Cryptosystem", Technical Report of IEICE of Japan, CPM99-124, ICD99-220, pp.3138, Dec (1999)

(16) T. Nakamura and H. Kasahara: "An Architecture of a HighSpeed Quasi-Parallel Divider for Numbers of Arbitrary Word Length", Trans. IEE of Japan, Vol.120-C, No.1, pp.158-167 (2000-1)

(17) Hwan, K: "Computer Arithmetic/Principles, Architecture, and Design", John Wily \& Sons, Inc. (1979)

Tsugio Nakamura (Member) received BE, ME and DE in

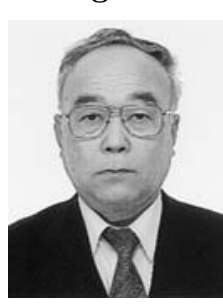
electrical engineering from Tokyo Denki University, Japan in 1971, 1990 and 1994, respectively. In 1971, he affiliated with Nippon Denki Seiki Co. Currently, he is an assistant professor in the Department of Information Network, Kokusai Junior College. His research interests include digital operation circuits, high-speed cryptosystems, surreptitious use prevention mechanisms for integrated circuit and hardware design technique for next generation ULSI. He is the author of The Foundations of Digital Circuit(Nihon Riko Shuppankai), Tecchnology of Digital Circuit Desighn -Example Collection of One-Chip Implementations(Nihon Riko Shuppankai), Introduction to Digital Circuit(Nihon Riko Shuppankai), Electronics Circuits(2)-Digital Circuits Edition(Corona) and other book. He is a member of IEEE Computer Society.
Kazuhiro Abe (Non-member) received BE and ME in elec-

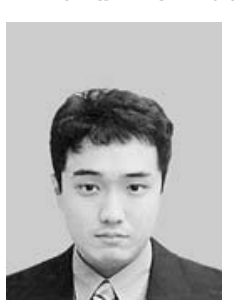
trical engineering from Tokyo Denki University, Japan in 1998 and 2000, respectively. He currently works at Fujitsu LTD., Tokyo from 2000. He is a member of I.E.I.C.E., Japan.

Narito Fuyutsume (Member) received BE, ME and DE in

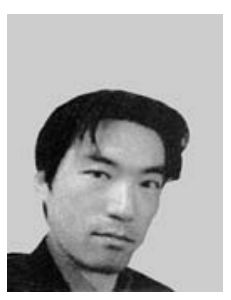
electrical engineering from Tokyo Denki University, Japan in 1995, 1997 and 2000, respectively. He is currently an assistant in the School of Information Environment, Tokyo Denki University. His research interest is computer networks. He is a member of I.E.I.C.E., Japan.

Hiroshi Kasahara (Member) received BE, ME and DE in

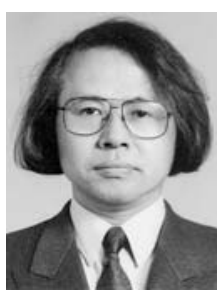
electrical engineering from Tokyo Denki University, Japan in 1964, 1967, 1970, respectively. He was a professor in the Department of Electrical Engineering, Tokyo Denki University to 2000. Currently, he is a professor in the School of Information Environment, at the same university. His research interests include power electronics, computer systems, distributed processing systems for control, design of object-oriented systems, interactive pulse diagnosis information processing systems and high-speed cryptosystems. He is a member of IEEE Computer Society, ACM, Inf. Proc. Soc., Japan and I.E.I.C.E., Japan.

Teruo Tanaka (Member) received BE and ME in electri-

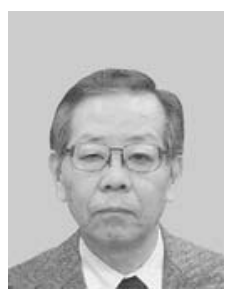
cal engineering from Tokyo Denki University, Japan in 1966 and 1969, respectively. He is currently a professor in the Department of Electrical Engineering, Tokyo Denki University. His research interest is power electronics. 